\title{
Una interpretación estructuralista de la leyenda del Don Juan ${ }^{1}$
}

El Don Juan, como personaje de la literatura, ha sido tema predilecto de críticos literarios porque, entre otras razones, es inquietante su larga existencia - tres siglos- a través de distintos lenguajes culturales, como el dramático, el narrativo, el poético, el operístico, etcétera. Asimismo, se le ha estudiado descle diferentes perspectivas, entre las que destaca la tematológica en que se analiza la evolución del Don Juan en la literatura a través de las distintas épocas (Singer, Weistein, De Bénotte, Baquero). También la psicología, la sociología, la filosofía y la antropología, entre otras disciplinas, han reflexionado sobre la controvertida figura del Don Juan, dado que ésta representa al rebelde, al seductor, al disidente, al profanador, al libertino, al blasfemo, al irredento, en una palabra, al hombre que encarna la trasgresión constante al orden social establecido.

A esa amplia bibliografía donjuanesca que interpreta, escudriña e historia la leyenda del Don Juan, se suma el estudio de Jean Rousset, ${ }^{1}$ el cual está orientado hacia el análisis del mito. A diferencia de los ensayos históricos, este autor no se interesa por la génesis del personaje, sino por las estructuras internas del mito que, según su opinión, son las que le han permitido subsistir en la literatura a lo largo de los siglos, y son al mismo tiempo las que, en un momento de desarticulación interna del sistema mítico, ha puesto en crisis la conti-

1 Jean Rousset, El mito del Don Juan, México, Fondo de Cultura Económi$\mathrm{ca}, 1985,267 \mathrm{pp}$. 
nuidad del Don Juan, nacido en España y conformado en el drama de Tirso de Molina.

El primer problema que enfrenta Rousset es saber si se puede llamar mito al relato del Don Juan. Desde el punto de vista de los etnólogos, aquellos que definen al mito por los contenidos y establecen que son las leyendas de origen las que se consideran como mitos, indudablemente que la leyenda del Don Juan no puede incluirse en esta clasificación. Tampoco entraría dentro de la concepción del mito de Eliade ni la de Lévi-Strauss; de este último, Rousset recoge únicamente la idea del anonimato de los mitos como uno de sus rasgos representativos; en el caso del Don Juan, su inicio lo tiene en la pieza teatral de Tirso, consecuentemente no podría considerársele como anónimo.

Pese a ello, Rousset señala otras características que le permiten fundamentar al Don Juan como un mito. En primer lugar, el contener elementos que representan la pervivencia de un fondo mítico y que afloran en el Don Juan, como la presencia del Difunto, sobre todo en el pasaje de la cena, que se relaciona con antiguos cultos a los muertos. En segundo término, el hecho de que, si bien el origen del mito se inicia en un autor en concreto, posteriormente, la leyenda sigue independientemente su trayectoria cultural y se olvida de su origen español; entonces, el mito pertenece a todos y a nadie: "reconocemos alli un rasgo propio del mito, su anonimidad ligada a su poder duradero sobre la conciencia colectiva".

Aunque estas justificaciones están débilmente sustentadas, dado que atienden a dos crtierios teóricos distintos, y porque de éstos el autor sólo toma parte de sus planteamientos (pervivencia y anonimato), sin embargo, considero que se valida Ia introducción del Don Juan en los linderos del mito porque Rousset, a través cle su análisis, establece una homología entre las estructuras internas que fundan los mitos (según LéviStrauss) y la microestructura de la leyenda del Don Juan; esto es, tanto el mito como el relato del Don Juan obedecen a un 
sistema interno de relaciones que puede ser explicado a través de los elementos que lo constituyen.

Según Lévi-Strauss, Ios mitos se forman "por unidades constitutivas" y dichas unidades o "mitemas" adquieren su función significativa al establecer relaciones y combinaciones entre sí. Estas relaciones constituyen, a su vez, un sistema que incluye la dimensión diacrónica y sincrónica; con estos dos niveles las unidades se ubican de acuerdo a la función que cumplen. (Antropologia estructural.)

El modelo narrativo del mito del Don Juan propuesto por Rousset corresponde al anterior planteamiento utilizado por Lévi-Strauss en la descripción de las estructuras míticas. Según Rousset los tres elementos (unidades) que constituyen el mito donjuanesco son: el Difunto, el grupo femenino y el héroe; estos son los elementos invariantes (concepto de Jakobson) que establecen una dimensión paradigmática. En cada texto particular, los elementos constantes se actualizan de distinta manera (variantes) y se combinan de manera diferente. Continuando con la perspectiva estructuralista, Rousset afirma que la ausencia de uno de estos elementos significa que se narra otra historia, porque un sistema mítico debe conservar las mismas invariantes; por otro lado, señala que la transformación de las relaciones entre los elementos o la alteración de uno ellos, modifica el sistema del mito y produce un cambio en el nivel de la significación.

Esa microestructura narrativa de tres elementos, permite a Rousset partir de un criterio coherente que explique la leyenda clel Don Juan como una estructura cultural significativa y le proporciona también una base teórica para elegir un corpus de textos literarios pertinentes a su investigación: sólo seleccionará aquellos en los que los tres elementos invariantes estén presentes. Por esta razón, deja de lado las versiones que no incluyen las apariciones del. Difunto, elemento primordial en la estructura del mito.

Según explica el autor, los tres elementos establecen entre sí un tipo de relaciones triangulares que permite flexibilidad y 
movilidad en el interior del mito; de aquí la posibilidad de combinarse de distinta manera en cada una de las versionzes. La movilidad interna del mito proviene de una red de fuerzas que se relaciọnan por "acción" y "reacción" y se constituyen en un "nudo de relaciones".

En esta microestructura narrativa, la función principal la cumplen el Difunto y su hija; se subordinan a ésta, tanto el héroe como los demás elementos. Sorprende que Rousset no destaque al Don Juan como el elemento principal de la leyenda, pero su explicación es que el mito del Seductor era, en su principio, un "mito de situación" y fue a partir del siglo xrx cuando se transforma en un "mito de héroe".

A partir de estos principios de análisis, Jean Rousset divide su trabajo en dos partes y un anexo en el que antologa trozos de textos importantes para su estudio. Se puede decir que el primer capítulo es un análisis intrasistémico, en el que se explican las particularidades, funciones y relaciones de los tres elementos invariantes. En el segundo apartado, se estudia la procedencia oral o literaria de los tres elementos, las variaciones de éstos al modificarse la estructura interna del mito, así como las transformaciones que sufren al pasar de un sistema dramático al operístico y al narrativo. De esta manera, el enfoque estructural inicial se amplía al considerar niveles extratextuales y también al tomar en cuenta criterios teóricos que definen los distintos discursos literarios.

El primer capítulo es el de mayor importancia porque ofrece un análisis de cada uno de los elementos constitutivos del mito y también porque sistematiza la clase de funciones que éstos cumplen, para finalmente establecer $\longrightarrow$ sin decirlo explícitamente- que son los cambios intrasistémicos los que producen las modificaciones en el nivel de la significación. De esta primera parte se extraen los comentarios más representativos que ejemplifican este tipo de estudio.

Para justificar el por qué considera el Difunto como el elemento más importante en la leyenda, Rousset dice que el Don Juan es la historia de "un culpable sometido a inculpación", 
pero que no se da cuenta de su estado hasta que el veredicto final del Difunto lo condena; por eso el desenlace de la historia es lo que marca definitivamente el relato y la suerte del héroe. De allí que el autor decida iniciar su estudio en el pasaje donde la historia termina: las apariciones del Difunto y la muerte del héroe.

Las escenas de las tres visitas del Convidado de Piedra ocurren, en dos de los casos, en lugares sagrados (el camposanto y la iglesia), donde lo inesperado o insólito puede ocurrir y donde se une el más allá con el más acá. Su condición de sagrados preanuncia la introducción de lo "fantástico y de la tragedia en la comedia". Sin embargo, hay textos que presentan variaciones de este espacio sagrado, que en algunos casos llega a desaparecer $y$, consecuentemente, también se elimina una de las características del mito donjuanesco: el enfrentamiento de la fuerza celestial y la terrena.

En el primer encuentro con la estatua del Comendador, el Don Juan lo reconoce y lo afrenta: "Sin esta ofensa al Difunto, la pieza se detendría y tomaría otra dirección". Esta acción es la que desata el desenlace trágico de la obra: la condena y muerte del Don Juan. Según Rousset, esta escena permite la introducción de lo fantástico y lo sobrenatural en la ficción vivida por los protagonistas de la comedia.

El segundo encuentro, ocurrido en la casa del don Juan, está formado por tres etapas: la cena del Don Juan, su interrupción por al entrada del Convidado de Piedra, la confrontación y contrainvitación. Cada una de ellas es susceptible de ser modificada o ampliada de acuerdo a las versiones distintas. Importantes son las variantes que introducen Bertati, Gazzaniga, Da Ponte y Mozart, porque unen el festín y el castigo sobrenatural, lo que "modifica profundamente el sentido del desenlace: la muerte cae en medio del placer".

El tercer enfrentamiento entre el Difunto y el Don Juan acontece en la iglesia cuando, en señal de un compromiso ineludible, el Comendador ordena: "Dame la mano", por ser el Difunto un enviado celestial que castiga al hereje por sus pe- 
cados. Sin embargo, este final es cambiado en algunos autores románticos como en Zorrilla, en cuya pieza, Inés, "Ana" redime a su amante. Al señalar esta modificación y de acuerdo con el criterio estructuralista de análisis, Rousset afirma: "Bas" te pues, el menor cambio en el desenlace para que el conjunto se modifique". Entonces todo el sistema donjuanesco girará y puede haber diversos desenlaces: se remplaza la estatua por un espíritu femenino, por un ser vivo, por un réprobo que se suicida, etcétera.

El grupo de víctimas seducidas por el héroe es el segundo elemento invariante que se estudia; éste tiene una gran importancia porque permite que se caracterice al Don Juan como un inconstante; la pluralidad femenina testimonia este rasgo. Dentro del grupo destaca Ana que funge como vínculo entre el seductor y el Difunto; precisamente por ser una figura de enlace, es necesario que se estudien sus posiciones y desplazamientos, tanto dentro del grupo femenino, como en relación con el Comendador y con el héroe.

Para explicar las variantes de doña Ana, Rousset continúa con el análisis estructural al oponer dos tipos de actualizaciones extremas de este personaje: la una que llama "elíptica", en la cual Ana está ausente y la otra, "hipertrófica", en la que prevalece la presencia de esta dama sobre las otras figuras femeninas. El primer caso corresponde a Molière, el segundo a Pushkin y Grabbe; en medio de ambos extremos hay una infinidad de soluciones intermedias.

Para Rousset, el modelo mejor organizado y el que le sirve de pauta para el estudio del grupo femenino es el de la pieza de Tirso. Allí, el grupo femenino está compuesto por dos damas nobles y dos del pueblo, lo que constituye una primera simetría por oposición, pero a esta simetría se opone el orden de aparición en escena de las víctimas, que se efectúa alternando las clases sociales a las que pertenecen; el grupo se polariza nuevamente porque difiere la forma de conquista de las damas nobles de la utilizada con las villanas. La coincidencia que las une es que todas ellas son engañadas $y$, salvo 
por momentos en los que una de ellas piensa estar enamorada, ninguna ama a Don Juan, y en cambio todas lo odian; además, el odio proviene del ultraje recibido y no de su amor defraudado. Este primer modelo femenino va a cambiar a través de las distintas versiones: las hay en que las víctimas son. presentadas como una multitud (Shadwell y Rostand) o son reducidas a un trío o a un dúo (Pushkin).

Hay que considerar otro grupo de víctimas que no aparece en el espacio escénico; éste pertenece a los relatos de aventuras amorosas tenidas anteriormente por el Seductor; en muchos casos, son catálogos de nombres recordados por el criado (Molière). Constituyen la masa anónima inmolada por Don Juan y utilizada para ganar prestigio ante los otros participantes de la comedia.

En el análisis de doña Ana, el autor simplifica las diversas versiones de este personaje en tres posibilidades: la primera es cuando esta figura femenina es privilegiada sobre el grupo femenino y termina por eliminarlo; entonces la pluralidad de mujeres se reduce y desaparece. Consecuentemente, la inconstancia del héroe se transforma en fidelidad (Zorrilla). Tal es la variante romántica, desconocida en los textos de los siglos xvir y xvir.

Opuestamente, el segundo modelo reduce a doña Ana a una existencia mínima (Molière), solución que es contraria a la lógica del sistema, pero que es posible encontrar y que produce un debilitamiento en la tensión entre el Comendador y Don Juan, porque deja de haber un motivo de enfrentamiento entre ambos personajes. Entre los dos polos, Rousset menciona una gama de versiones que mantienen distintos nexos entre el Convidado de Piedra y el Seductor.

La hegemonía o desaparición de la figura de doña Ana, modifica "en un sentido u otro el equilibrio del grupo femenino", las características del Don Juan y las relaciones con el Comendador.

Entre las versiones importantes está un texto anónimo italiano L'Atiesta Fulminato, en éste, la figura femenina es rap- 
tada de un convento, se enamora de Don Juan, pero es rechazada y muere extenuada por la penitencia. También está la variante de Molière y las posteriores de Hoffmann y Mozart. Estas dos últimas otorgan al mito una nueva vitalidad y sentido, "nace el Don Juan romántico, el Don Juan puesto en presencia de Ana", para ser redimiclo por el amor. De esta manera, doña Ana desplaza al Comendador en su función de sentenciar al Seductor, pero no para condenarlo sino para salvarlo; es entonces cuando el Difunto pasa a un segundo término y luego desaparece.

En el desenlace reinventado por Zorrilla, sin dejar de entroncar con el problema teológico de Tírso, el héroe será objeto de la gracia, con la condición de que ame y que lo ame una mujer, que ella muera por él y arriesgue su salvación espiritual por la salvación del pecador. La figura de doña Ana, la más borrosa en el grupo femenino de Tirso, se transforma en omnipotente: subordina al padre y al Seductor. Este desequilibrio en las relaciones de los elementos del mito, hará que posteriormente estalle esta figura femenina y sea el inicio del término del mito.

Por las causas anteriores, en textos contemporáneos aparece una Ana que de perseguida se convierte en perseguidora (Shaw); la Ana del modelo primario se extingue porque se disloca el sistema de relaciones interno del mito, $y$, así como en algunas versiones desaparece el Difunto, Ana se transforma en una mujer como las demás.

El tercer y último componente del mito -el héroe- es estudiado en tres aspectos que son agrupados en parejas de opuestos, tales como el réprobo y sus jueces, el comediante y sus espectadores y el improvisador ante la permanencia.

Las relaciones entre el Pecador y sus jueces cambian de acuerdo con las modificaciones que sufren los elementos de esta oposición en las distintas variantes. En la pieza de Tirso, el delito cometido por el Don Juan corresponde a un nivel celestial, por profanar los lugares sagrados y rechazar con dureza. las oportunidades de arrepentimiento; no tanto por traicionar 
mujeres, amigos, príncipes y familiares. En la época del Romanticismo, el Seductor entra en un proceso de laicización y el Libertino deja de ser un transgresor de leyes divinas, para ser únicamente de las terrenas. Esta transformación termina en. nuestros días, cuando el Don Juan no es considerado pecador, ni delincuente, cuando se le reduce al papel de seductor $y$, como tal, "dador del placer y el festín".

La segunda relación es la que se establece entre el Burlador, en su papel de comediante, frente a los demás personajes. En el teatro - -explica Rousset-, se abren dos espacios escénicos, el del silencioso espectador pasivo que está en la sala y el otro que se encuentra en el interior del escenario entre el Comdiante y las víctimas de su seducción. Este segundo espacio. es el que Rousset considera significativo, porque es en donde el Burlador hace gala de diversos subterfugios para engañar a sus. espectadores o víctimas; entre los recursos utilizados, el de mayor importancia, por su riqueza, es el embeleso por medio de la palabra. En este juego de la seducción y la fascinación, sus. víctimas son los espectadores de sus encantos verbales; en las. distintas versiones, las víctimas tienen respuestas que van descle el creer las fantasías verbales del Burlador hasta el no creerlas.

Este juego verbal se subvierte en la literatura del siglo XIX y $\mathrm{xx}$, porque el Don Juan ha transformado su deseo de engañar, si bien continúa siendo inconstante, "pero ama cada vez que trata de seducir. Lo que equivale a decir que, mal comediante, cae en su propio juego" (Rostand y Frisch). Ante la desarticulación del sistema de relaciones en la época actual, el Comediante es reducido a la condición de títere o de un intelectual anarquizante o de un narciso inepto para el amor.

El último par de oposiciones que estudia Rousset es la improvisación frente a la permanencia; la primera categoría corresponde al Don Juan quien vive en un presente sin memoria del pasado ni con proyecto hacia el futuro y actúa de acuerdo con lo que le ofrece el azar; opuestamente, la segunda categoría corresponde al grupo femenino que representa la fidelidad al compromiso. El conflicto entre ambos términos se sus- 
cita cuando el "paladín de lo instantáneo" goza del instante de la seducción, pero carece del pasado y del futuro, y por lo tanto no puede cumplir sus promesas de matrimonio. También contrario a lo fugitivo del Don Juan, está la totalidad permanente de la estatua animada, y es el Difunto el que pone término a las idas y venidas del "perito en metamorfosis". Por eso, el desenlace de la pieza de "Tirso asegura el principio de coherencia del mito, el símbolo de lo permanente aplasta al "hombre de viento". Al referirse a las variantes en las cuales Don Juan se salva por intermedio del amor, Rousset comenta que es entonces cuando el inconstante es curado de su amnesia y reconoce sus actos pasados. "Salvar al Don Juan es arrancarlo de su pasión por lo instantáneo".

En la segunda parte del trabajo, el mayor interés está en la explicación de los cambios que sufre la estructura del mito al pasar a distintos sistemas semióticos, como son el dramático, el de la commedia dell'arte, el de la ópera y el narrativo.

En esta parte de la investigación, Rousset sustenta su análisis en el supuesto de que cada modelo discursivo (dramático, bufo, operístico y narrativo) tiene constantes genéricas que le otorgan unidad a cada uno de ellos; ahora bien, estas caracteríticas tipológicas influyen sobre la estructura del mito y lo transforman de acuerdo con el modelo discursivo correspondiente. A su vez, el sistema del mito también va a incidir sobre estos sistemas discursivos.

De los casos estudiados se destacan, por su interés, el de la commedia dell'arte, cuyo espíritu cle comedia desnaturaliza la dimensión religiosa del drama. Por otro lado, está el modelo operístico que también influye por medio de los dúos, tríos, cuartetos y solos, los que provocan que la febril rapidez de la historia se detenga; la acción se vuelve lenta o se inmoviliza así se crea un nuevo ritmo en la representación. En el nivel del argumento, hay un cambio importante, al desplazarse la muerte del Comendador a las primeras escenas (Mozart). Por último, el finale espectacular de la ópera modifica el término 
de la historia y lo presenta como un contraste entre la fiesta y la condenación.

El cambio del sistema dramático al narrativo implica otras modificaciones, tales como el tener un narrador intratextual que relata el espectáculo musical del Don Juan (Hoffmann, Sand, Jouhandeau, Des Forés); el que haya un destinatario inserto o no en el texto; el que se rompa el orden temporal de los acontecimientos relatados y, en lugar de la "polifonía de los códigos dramáticos", se exprese una monodia textual.

En la época contemporánea, el Don Juan conserva sólo algunos de su atributos, como el gozador voluptuoso, el hombre del placer, en suma, el ser del deseo sexual; limitar las posibilidades del héroe es estar escribiendo otra historia $y$, aunque la vida del mito se sustenta en sus metamorfosis, los cambios deben producirse dentro del sistema fundador. Por eso, las variaciones del mito del Don Juan, en la época actual, hacen suponer que se está desintegrando su figura al convertirse en virilidad indecisa (Marañón), miedo a la mujer que lo persigue (Shaw, Frisch), parálisis narcisista o preferencia por su propio sexo; es también el libertino, perverso, "atravesado por la sombría locura del sexo" o impulsado "por la fuga de la contranatura" (Foucault).

En este callejón sin salida, el mito del Don Juan parece estar condenado a desaparecer. Sin embargo, comenta optimistamente Rousset, en cuestiones culturales los tiempos cambian y, si estuvo a punto de morir en el siglo xvir, "podría renacer, una vez más. Sólo haría falta un nuevo Mozart".

Es indudable que, bajo el ameno y bien documentado estudio del Don Juan, están algunas propuestas estructuralistas que organizan el análisis; el no explicitarlas en farragosa argumentación es una de las cualidades de este trabajo; el autor las asume como un sustrato cultural, ya de suyo conocido por la crítica literaria, y las emplea con fina destreza. Como se ha hecho notar, el análisis de Rousset no se queda en el esquema metodológico, sino que, a partir de éste -que sirve para sistematizar las versiones utilizadas- comenta y explica 
las características que el mito adquiere en cada una de las variantes estudiadas.

Sin embargo, hay que señalar una observación al trabajo de Rousset: según el método utilizado, todo su estudio conlleva un supuesto cuestionable: el considerar que los cambios ocurridos en el mito del Don Juan sólo son producto tanto de las alteraciones que sufren los elementos dentro del sistema donjuanesco, como de los cambios ocurridos al pasar de un discurso dramático a otros tipos de discursos. Nada se dice de lo que origina estas transformaciones y el por qué se producen ren una época y no en otra; en suma, nada se dice del entorno :sociocultural que está determinando todos los productos culturales, y en este caso, la literatura. Naturalmente, contestar a estas inquietudes llevaría al autor a otros terrenos que él desde un principio no se propuso andar. 